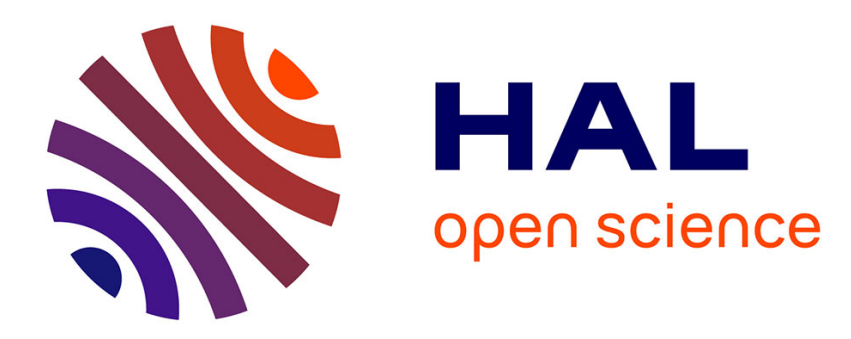

\title{
Quelques propriétés électrochimiques du niobium
}

Jacques Pagetti, Jean Talbot

\section{To cite this version:}

Jacques Pagetti, Jean Talbot. Quelques propriétés électrochimiques du niobium. Revue de Physique Appliquée, 1970, 5 (3), pp.365-369. 10.1051/rphysap:0197000503036500 . jpa-00243403

\section{HAL Id: jpa-00243403 https://hal.science/jpa-00243403}

Submitted on 1 Jan 1970

HAL is a multi-disciplinary open access archive for the deposit and dissemination of scientific research documents, whether they are published or not. The documents may come from teaching and research institutions in France or abroad, or from public or private research centers.
L'archive ouverte pluridisciplinaire HAL, est destinée au dépôt et à la diffusion de documents scientifiques de niveau recherche, publiés ou non, émanant des établissements d'enseignement et de recherche français ou étrangers, des laboratoires publics ou privés. 


\title{
QUELQUES PROPRIÉTÉS ÉLECTROCHIMIQUES DU NIOBIUM
}

\author{
par Jacques PAGETTI et Jean TALBOT \\ Laboratoire de Génie Chimique. Ecole Nationale Supérieure de Chimie de Paris, \\ 11, rue Pierre-et-Marie-Curie, Paris $5^{\mathrm{e}}$
}

\begin{abstract}
Résumé. - Le comportement du niobium dans les solutions acides et alcalines a été étudié par des méthodes électrochimiques.

Afin d'obtenir un état de surface bien défini nous avons mis au point une méthode de polissage électrolytique du niobium.

La tenue remarquable du niobium dans les acides à l'exception de l'acide fluorhydrique, peut être attribuée à la formation d'un film d'oxyde protecteur.

Dans le milieu alcalin la vitesse de corrosion du métal dépend de la concentration du réactif d'une part et de la teneur en oxygène dissous d'autre part.
\end{abstract}

\begin{abstract}
Corrosion of niobium in various acid and alkaline media, at room temperature, has been studied using electrochemical methods.

An electrolytic polishing method is developed in order to obtain a well defined reproductible surface.

The good resistance of niobium in acidic solutions, except fluorhydric acid, has been attributed to a protective film of niobium oxide.

Preliminary results have been obtained on the anodic behaviour of niobium in deaerated sodium hydroxide. In addition, the effect of dissolved oxygen on the corrosion of niobium has been examined.
\end{abstract}

La lecture du programme de ces journées montre à l'évidence que les recherches actuellement entreprises sur la corrosion du niobium sont pratiquement toutes consacrées à l'étude des propriétés réfractaires de ce métal.

Si nous avons entrepris une étude du comportement du niobium en solution, c'est que nous avons constaté que les caractéristiques chimiques ou électrochimiques du niobium métallique sont encore bien mal connues. La bibliographie $[1,2,3,4,5,6]$ fait bien état de la faible réactivité du niobium en milieu acide et d'une susceptibilité plus grande en milieu alcalin. Mais il s'agit le plus souvent de résultats assez fragmentaires et d'un caractère très qualitatif.

Je me propose donc ici d'indiquer les résultats que nous avons obtenus en utilisant les méthodes électrochimiques employées de façon courante pour l'étude de la corrosion des métaux en solution.

Il me paraît utile tout d'abord de rappeler très rapidement le principe de ces méthodes.

I. Mesure du potentiel de dissolution. Mesure de l'intensité du courant de corrosion. - Lorsqu'un métal est plongé dans un électrolyte, il a tendance à passer en solution sous forme d'ions positifs tandis que les charges négatives apparaissent à sa surface. Il se forme ce que l'on appelle une double couche, que l'on peut assimiler à un condensateur, et dont la capacité peut être déterminée. Mais il s'agit là d'une mesure délicate. Le plus souvent, on se contente de mesurer la différence de potentiel qui existe entre le métal et une électrode de référence, par exemple au calomel saturé, plongée dans le même électrolyte. Afin d'éliminer les phénomènes de polarisation qui se produisent lorsqu'un courant passe dans le circuit ainsi établi, on utilise pour la mesure un millivoltmètre électronique de très forte impédance d'entrée. Plus le potentiel est positif, ou noble, et plus le métal a tendance à résister à l'action du réactif. Ce potentiel est appelé potentiel de dissolution ou de corrosion.

A ce potentiel, deux réactions se produisent à la surface du métal : une réaction anodique d'oxydation du métal et une réaction cathodique qui, suivant la nature et le $\mathrm{pH}$ de la solution, peut être par exemple la réduction de l'hydrogène, de l'oxygène ou de l'eau. L'intensité de chacune de ces réactions est égale en valeur absolue ; c'est le courant d'échange ou courant de corrosion.

$\mathrm{Si}$, en introduisant une électrode auxiliaire en platine, on fait varier le potentiel appliqué à l'électrode étudiée, potentiel que l'on repère par rapport à une électrode de référence, l'intensité du courant des deux réactions varie et il est possible de tracer ce que l'on appelle une courbe globale intensité-potentiel qui est la somme des deux courbes élémentaires anodique et cathodique qui elles ne sont pas accessibles à l'expérience. A partir de cette courbe globale, la détermination de l'intensité de corrosion peut être faite de façon 
aisée. Un schéma simple (Fig. 1) montre que si la pente des courbes élémentaires est grande, l'intensité du courant de corrosion est élevée. De plus, la position relative des courbes élémentaires détermine l'intensité $\mathrm{du}$ courant de corrosion.

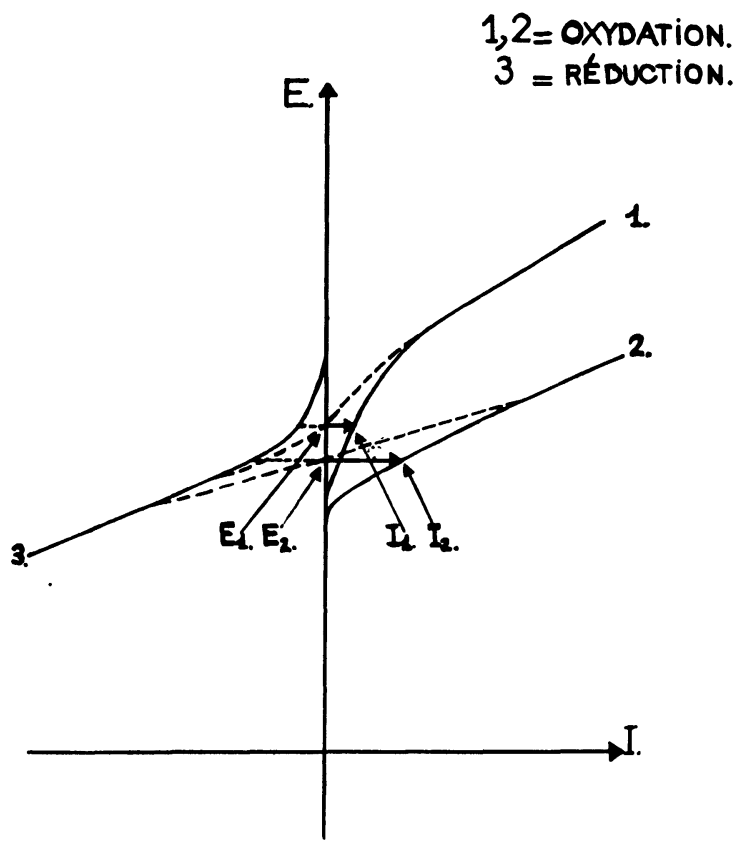

FIg. 1. - Influence de la pente des courbes élémentaires sur la vitesse d'attaque.

II. Etude électrochimique du niobium en milieu acide. - La deuxième figure indique le comportement du niobium dans différents acides. Il ne s'agit

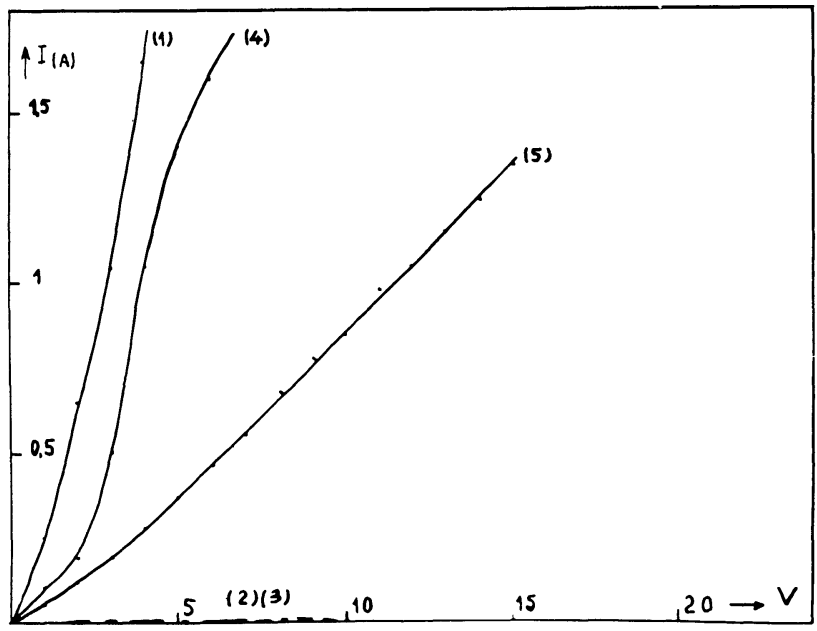

FIG. 2. - Courbes intensité-potentiel.

1 Acide fluorhydrique.

2 Acide sulfurique pur.

3 Acide lactique.

4 Acide fluorhydrique $40 \%$, acide sulfurique $60 \%$

5 Acide fluorhydrique $40 \%$, acide lactique $60 \%$.

pas à proprement parler de courbes intensité-potentiel telles que nous les avons précédemment définies. On mesure simplement dans ce cas l'intensité du cou- rant qui circule entre le niobium placé à l'anode et une cathode en graphite, en fonction de la tension appliquée aux bornes. L'attaque par l'acide fluorhydrique est rapide [1] ainsi que le mélange acide fluorhydrique $40 \%$ acide sulfurique $60 \%$ [4]. Elle est nulle dans l'acide sulfurique pur [2] et l'acide lactique pur [3]. L'addition d'acide lactique à l'acide fluorhydrique [5] ralentit nettement l'attaque du niobium. Il y a donc une véritable passivation du niobium dans certains acides.

Nous avons pensé qu'il serait peut être possible d'effectuer le polissage électrolytique du niobium en utilisant un bain d'acide fluorhydrique additionné de ralentisseurs tels que l'acide sulfurique et l'acide lactique. On sait que, selon Jacquet, pour obtenir un polissage électrolytique, il est nécessaire que la

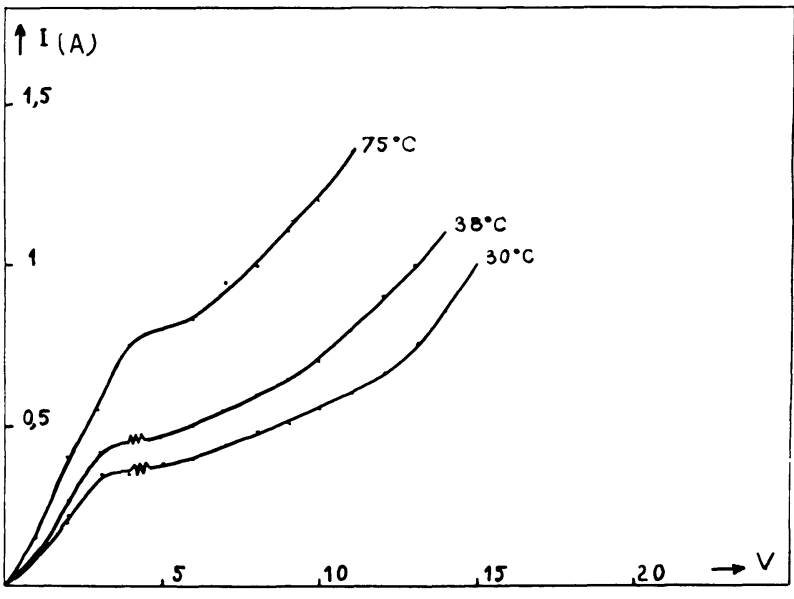

Fig. 3. - Influence de la température sur la forme des courbes $I=f(\mathrm{~V})$.

courbe $I=f(V)$ présente un palier d'intensité dans un intervalle de potentiels suffisamment étendu. La figure 3 indique les résultats que nous avons obtenus dans le mélange contenant $28 \%$ d'acide fluorhydrique, $36 \%$ d'acide sulfurique et $36 \%$ d'acide lactique. Un palier s'étendant sur 2 ou 3 volts apparaît lorsque la température du bain est comprise entre 35 et $40^{\circ} \mathrm{C}$. C'est dans ces conditions de température que nous avons obtenu les meilleurs résultats, pour une tension de 5,5 V entre les électrodes. La présence d'oscillations sur le palier vers $4 \mathrm{~V}$ permet de procéder à une attaque micrographique. La figure 4 montre, au grossissement 80 , l'aspect du niobium poli électrolytiquement et attaqué dans le bain sous tension.

Avec des échantillons ainsi polis, nous avons étudié le comportement du niobium dans différents acides par la méthode des courbes intensité-potentiel. Quel que soit l'acide utilisé, à l'exception de l'acide fluorhydrique, il se produit une passivation du métal, et l'intensité correspondant au palier est au plus égale à $2 \mu \mathrm{A} / \mathrm{cm}^{2}$, ce qui est très faible. L'intensité de corrosion est donc de cet ordre de grandeur. La figure 5 montre les résultats obtenus dans le cas de l'acide sulfurique (courbe 1). 


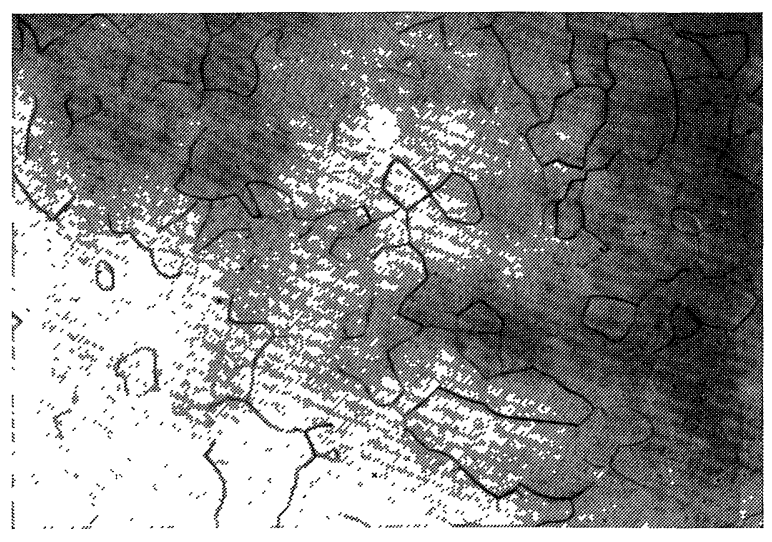

Fig. 4. $-(\times 80)$ Niobium poli électrolytiquement et attaqué 30 secondes dans le bain de polissage sous une tension de 4 volts.

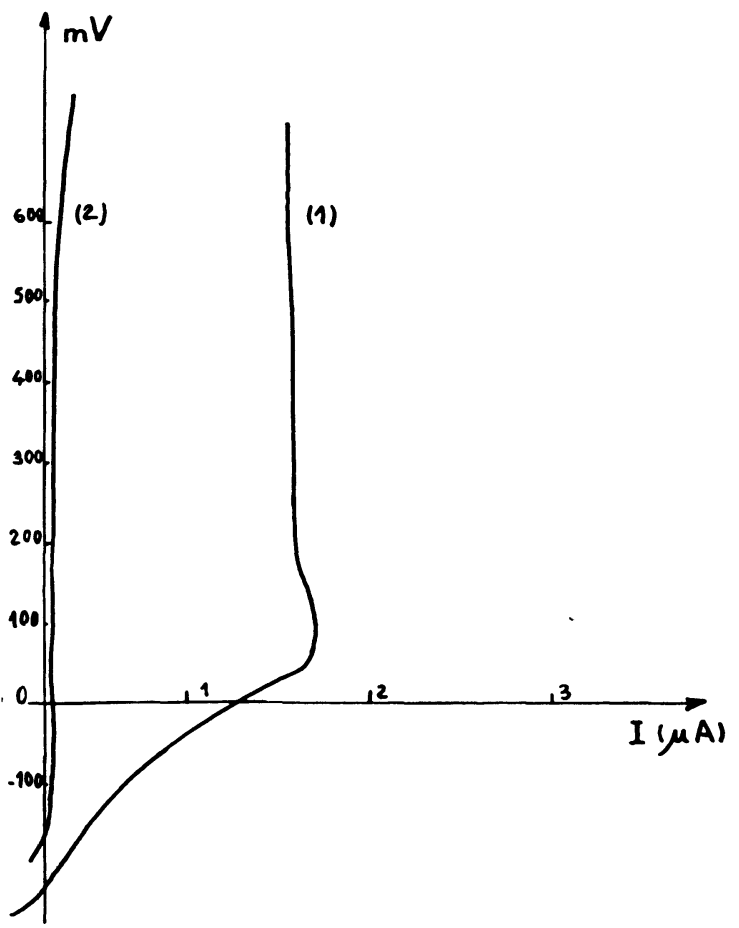

FIG. 5. - Aspect des courbes potentio-cinétiques relatives à des éprouvettes de niobium plongées dans les différents acides étudiés.

Le film d'oxyde vraisemblablement $\mathrm{Nb}_{2} \mathrm{O}_{5}$ qui se forme sur le niobium est donc étanche et très résistant. En effet, si on trace la courbe intensité-potentiel d'un échantillon préalablement polarisé anodiquement jusqu'à $+700 \mathrm{mV} / \mathrm{e} . \mathrm{c} . \mathrm{s}$, on observe que l'intensité correspondant au palier n'est qu'une fraction de $\mu \mathrm{A} / \mathrm{cm}^{2}$ (courbe 2).

L'attaque du niobium par les acides, exception faite de l'acide fluorhydrique, est donc très faible. $L$ 'aspect micrographique du métal reste inchangé même après une immersion prolongée des échantillons. La pureté du métal ne semble pas avoir d'influence ni la présence d'oxygène dans la solution. Le potentiel de dissolution se fixe très lentement, 48 heures environ, et sa valeur n'est pas très reproductible. On pourrait donner une interprétation électrochimique à ce phénomène, mais cela sortirait du cadre de cet exposé.
III. Etude électrochimique du niobium en milieu basique. - Les expériences ont été faites dans la soude, à différentes concentrations, en l'absence ou en présence d'oxygène dissous. Les réaction élémentaires qui peuvent intervenir sont les suivantes :

réaction anodique : oxydation du niobium

réactions cathodiques : réduction de l'eau réduction de l'oxygène.

C'est la position relative des courbes élémentaires qui conditionne le comportement du niobium.

a. SOLUTIONS DÉSAÉRÉES (Fig. 6). - La réactivité du niobium dépend de la concentration en soude de

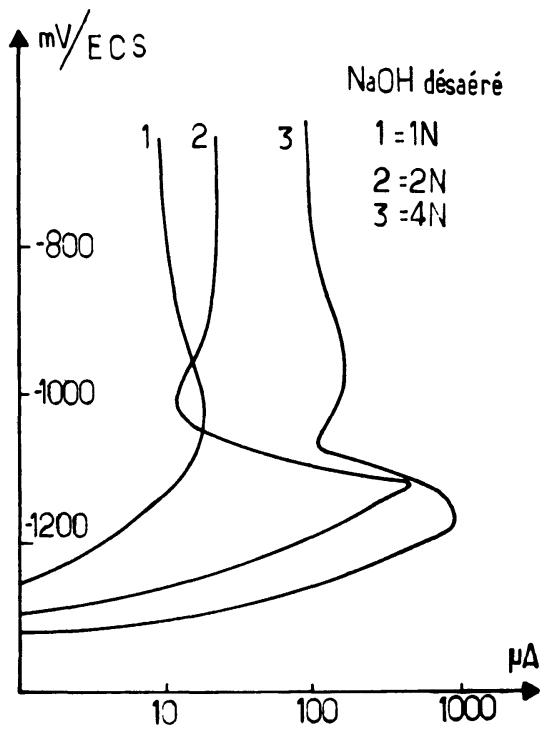

FIG. 6. - Courbes de polarisation anodique relatives à une éprouvette de niobium dans des solutions de soude désaérées.

la solution. Si la solution est normale, la pente de la courbe anodique est faible et l'intensité correspondant au palier de passivation est de l'ordre de $8 \mu \mathrm{A} / \mathrm{cm}^{2}$. Pour une solution $2 \mathrm{~N}$ ou $4 \mathrm{~N}$, on observe l'existence d'un pic d'activité qui précède la formation du palier. L'intensité correspondant au maximum du pic atteint alors environ $1000 \mu \mathrm{A} / \mathrm{cm}^{2}$ et la pente des courbes au voisinage du potentiel de dissolution est notablement plus grande que celle de la courbe tracée dans la solution normale. Il y a donc une augmentation de la vitesse d'attaque du niobium lorsque la concentration en soude de la solution croît.

b. SOlUTIONS SATURÉES EN OXYGÈNE. - La figure 7 montre les courbes intensité-potentiel obtenues dans une solution $4 \mathrm{~N}$ en milieu désaéré (courbe 1), dans une solution saturée en oxygène (courbe 2) et dans une solution contenant de l'eau oxygénée (courbe 3). On observe que le potentiel de dissolution devient de plus en plus positif quand on ajoute de l'oxygène et que la pente des courbes au voisinage du potentiel de dissolution croît parallèlement. Le palier qui apparaît sur la courbe relative au niobium plongé dans la solution contenant de l'eau oxygénée n'est plus un 


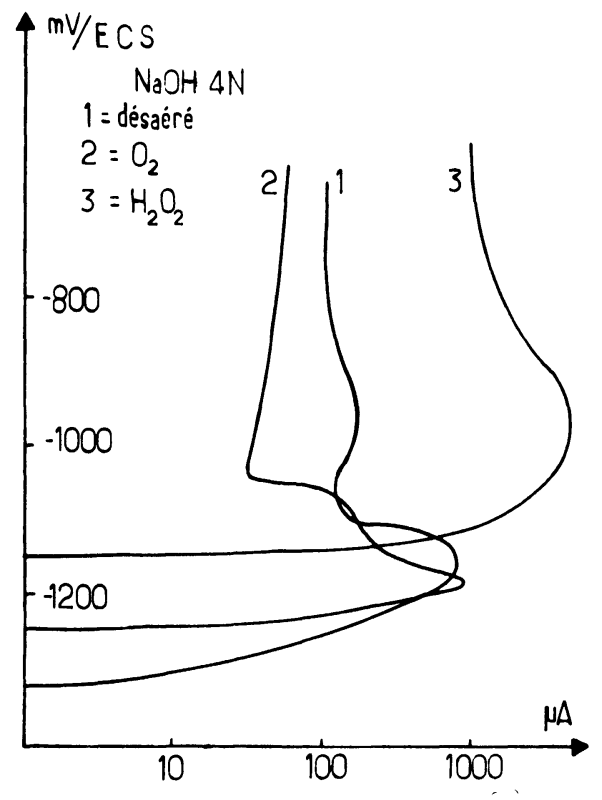

Fig. 7. - Courbes de polarisation anodiques relatives à une éprouvette de niobium dans une solution de soude $4 \mathrm{~N}$, désaérée, aérée et en présence de $\mathrm{H}_{2} \mathrm{O}_{2}$.

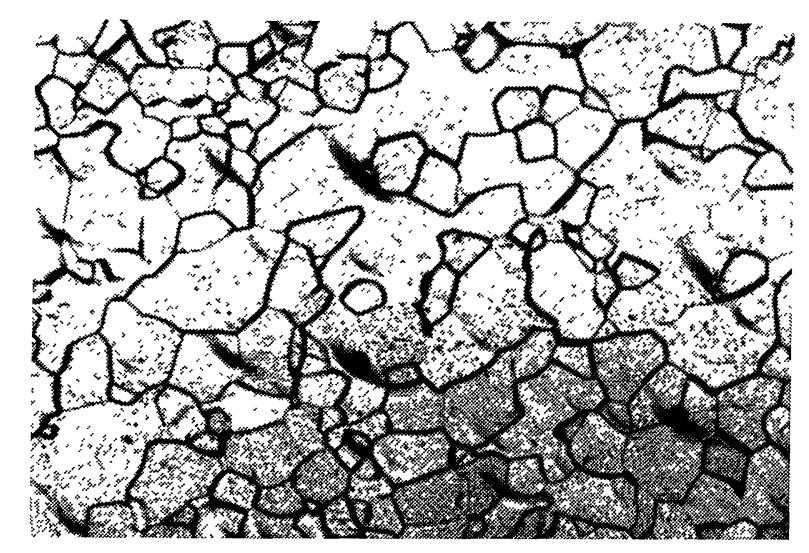

FIG. 8. $-(\times 200)$ Micrographie d'une éprouvette de niobium immergée pendant 1 heure dans une solution de soude $4 \mathrm{~N}$ en présence de $\mathrm{H}_{2} \mathrm{O}_{2}$.

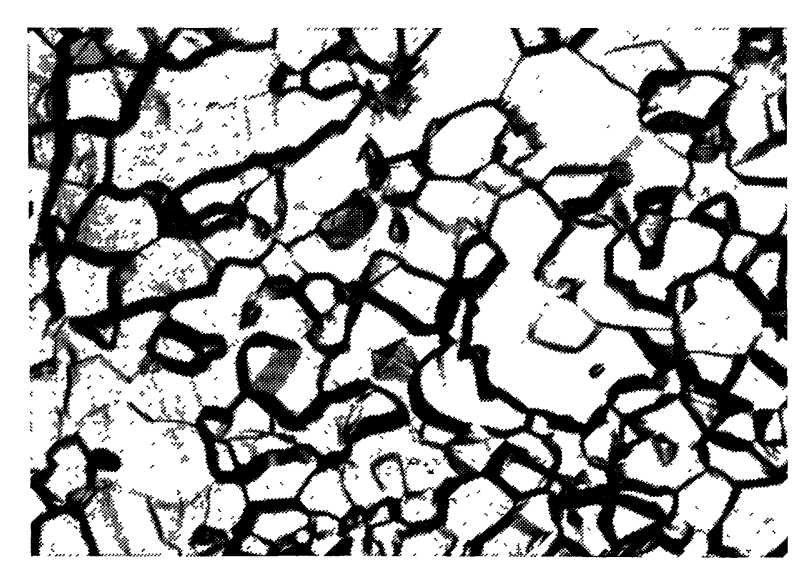

FIG. 9. - $(\times 200)$ Micrographie d'une éprouvette de niobium immergée pendant 7 heures dans une solution de soude $4 \mathrm{~N}$ en présence de $\mathrm{H}_{2} \mathrm{O}_{2}$. palier de passivation puisque l'intensité qui lui correspond est de l'ordre du milliampère par centimètre carré.

Il y a donc attaque du niobium aussi bien en présence d'oxygène qu'en présence d'eau oxygénée.

Les figures 8 et 9 montrent l'aspect du métal après immersion respectivement de 1 heure et de 7 heures dans la soude $4 \mathrm{~N}$ contenant de l'eau oxygénée $(\times 200)$.

IV. Interprétation des phénomènes. - Nous ne parlerons que des phénomènes de corrosion en milieu basique.

La courbe élémentaire anodique en milieu basique désaéré présente un pic d'activité dont la hauteur dépend de la concentration de la solution en soude. Il faut indiquer que la seule réaction cathodique qui intervient est la réaction de l'eau.

Lorsque l'on passe d'une solution normale à une solution $4 \mathrm{~N}$, la pente de la courbe intensité-potentiel dans le domaine anodique augmente et le potentiel de dissolution se déplace vers des valeurs plus négatives (Fig. 6).

Le schéma de la figure 10 permet d'interpréter les phénomènes observés. On constate que le déplacement du potentiel de dissolution vers des valeurs plus négatives par suite du déplacement de la courbe élémentaire anodique entraîne une augmentation de l'intensité du courant de corrosion.

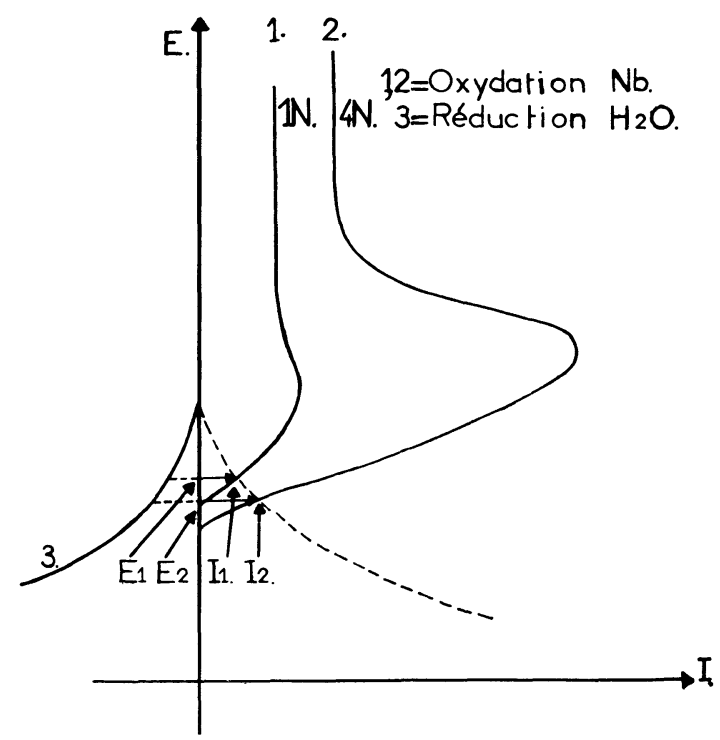

FIG. 10. - Positions relatives des courbes élémentaires dans le cas des solutions de soude $1 \mathrm{~N}$ et $4 \mathrm{~N}$ désaérées.

En milieu basique contenant de l'oxygène, la courbe élémentaire anodique présente la même allure qu'en l'absence d'oxygène dissous. Mais il convient alors de considérer deux courbes élémentaires cathodiques, réduction de l'eau et réduction de l'oxygène ou de l'eau oxygénée. Le schéma de la figure 11 montre que lorsque l'on passe d'une solution désaérée à une solution contenant de l'oxygène, d'une part le potentiel de 


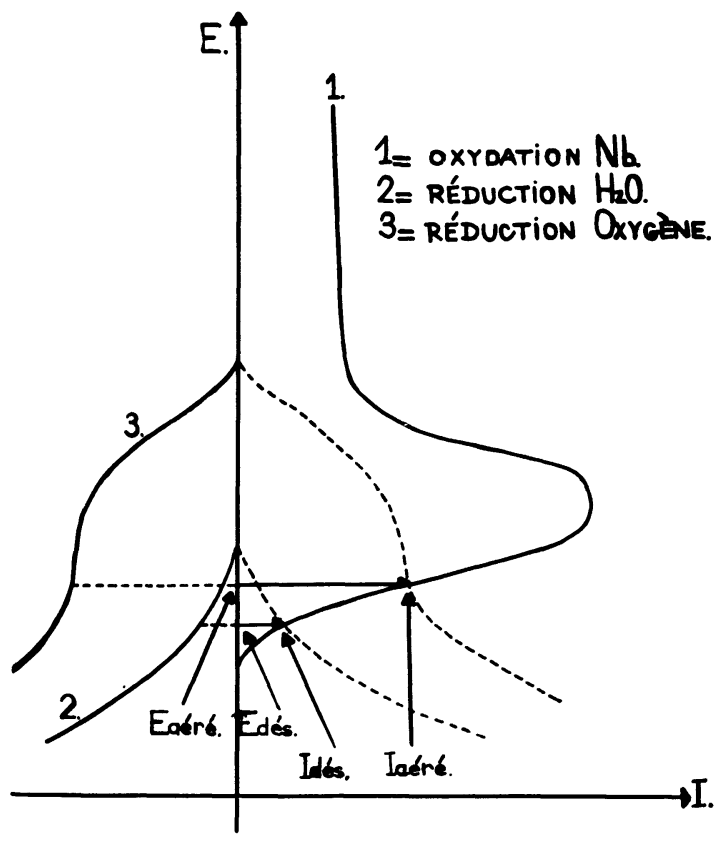

Fig. 11. - Positions relatives des courbes élémentaires dans le cas des solutions de soude désaérées et des solutions de soude aérées.

dissolution devient plus noble et d'autre part l'intensité du courant de corrosion croît. L'augmentation de la vitesse d'attaque dépend évidemment de la teneur de la solution en oxygène.

Il est intéressant de signaler, qu'en solution désaérée, le passage d'un potentiel plus noble à un potentiel moins noble, quand la concentration de la solution en soude croît, se traduit par une augmentation de l'intensité du courant de corrosion alors que le passage d'un potentiel plus noble à un potentiel moins noble quand on diminue la teneur en oxygène dissous se traduit par une diminution de l'intensité du courant de corrosion.

Nous ne connaissons pas la nature de la couche qui se forme à la surface du niobium en solution basique. Les premières études faites par rayons $\mathrm{X}$ et diffraction des électrons ne nous ont pas permis de la déterminer. Cependant, nous pensons qu'il ne s'agit pas d'un oxyde pur. Il est possible que la nature de la solution basique intervienne dans la composition du produit de corrosion. Nous poursuivons actuellement l'étude de ce composé.

Les recherches que nous avons faites sur le comportement du niobium en milieu acide et en milieu alcalin nous ont permis de montrer la très faible réactivité de ce métal en milieu acide, à l'exception de l'acide fluorhydrique. Cependant, l'attaque du niobium par HF peut être mise à profit pour le polissage électrolytique de sa surface.

En milieu basique, le niobium est attaqué à une vitesse qui augmente avec la concentration du réactif. La présence d'oxygène dissous dans la solution accélère l'attaque du métal. Il est possible de donner une interprétation électrochimique aux phénomènes observés.

\section{Bibliographie}

[1] Pascal, Nouveau traité de Chimie Minérale, 1958, XII, 360.

[2] DraPER, Electrochemica Acta, 1963, 11, 847.

[3] Draper, Harvey (J.), Acta Metallurgica, 1963, II, 873.

[4] Tingley (I. I.), Rogers (R. P.), Corrosion, 1969, 21, 167.
[5] Piron, Nobe (Ken), Corrosion, 1969, 25, 67.

[6] PourbaIX, Atlas d'équilibres électrochimiques, 1963, 248.

[7] Pagetti (J.), Talbot (J.), Corrosion Traitements Protection, 1967, 15. 